\title{
Genome-wide analysis of DNA methylation and risk of cardiovascular disease in a Chinese population
}

Yan Gao ${ }^{1 \dagger}$, Huifang Pang ${ }^{1 \dagger}$, Bowang Chen ${ }^{1}$, ChaoQun Wu' ${ }^{1}$, Yanping Wang ${ }^{1}$, Libo Hou' ${ }^{1}$, Siming Wang ${ }^{1}$, Dianjianyi Sun ${ }^{2}$ and Xin Zheng ${ }^{1 *}$

\begin{abstract}
Background: Systemic studies of association of genome-wide DNA methylated sites with cardiovascular disease (CVD) in prospective cohorts are lacking. Our aim was to identify DNA methylation sites associated with the risk of CVD and further investigate their potential predictive value in CVD development for high-risk subjects.

Methods: We performed an epigenome-wide association study (EWAS) to identify CpGs related to CVD development in a Chinese population. We adopted a nested case-control design based on data from China PEACE Million Persons Project. A total of 83 cases who developed CVD events during follow-up and 83 controls who were matched with cases by age, sex, BMI, ethnicity, medications treatment and behavior risk factors were included in the discovery stage. Genome-wide DNA methylation from whole blood was detected using Infinium Human Methylation EPIC Beadchip (850 K). For significant CpGs [FDR(false discovery rate) <0.005], we further validated in an independent cohort including 38 cases and 38 controls.
\end{abstract}

Results: In discovery set, we identified 8 significant CpGs (FDR <0.005) associated with the risk of CVD after adjustment for cell components, demographic and cardiac risk factors and the first 5 principal components. Two of these identified CpGs (cg06901278 and cg09306458 in UACA) were replicated in another independent set $(p<0.05)$. Enrichment analysis in 787 individual genes from 1036 CpGs in discovery set revealed a significant enrichment for anatomical structure homeostasis as well as regulation of vesicle-mediated transport. Receiver operating characteristic (ROC) analysis showed that the model combined 8 CVD-related CpGs with baseline characteristics showed much better predictive effect for CVD occurrence compared with the model with baseline characteristics only [AUC (area under the curve $)=0.967,95 \% \mathrm{Cl}(0.942-0.991) ; \mathrm{AUC}=0.621,95 \% \mathrm{Cl}(0.536-0.706) ; p=9.716 \mathrm{E}-15]$.

Conclusions: Our study identified the novel CpGs associated with CVD development and revealed their additional predictive power in the risk of CVD for high-risk subjects.

Keywords: EWAS, DNA methylation, CVD

*Correspondence: xin.zheng@fwoxford.org

†Yan Gao and Huifang Pang: Co-first author

${ }^{1}$ National Clinical Research Center for Cardiovascular Diseases, State

Key Laboratory of Cardiovascular Disease, Chinese Academy of Medical

Sciences and Peking Union Medical College, Fuwai Hospital, National

Center for Cardiovascular Diseases, 167 Beilishi Road, Beijing 100037,

People's Republic of China

Full list of author information is available at the end of the article

\section{Introduction}

Cardiovascular disease (CVD), a complex disease that is attributed to the interaction between environmental and genetic factors, is the leading cause of death in most countries $[1,2]$. It is necessary to elucidate the underlying biological mechanisms of CVD to enable early diagnosis and treatment of the disease. 
For the development of CVD, it is widely accepted that age, sex, high blood pressure, smoking, dyslipidemia, and diabetes are the major risk factors. Furthermore, over the past few years, many genome-wide association studies (GWAS) have identified relevant genetic polymorphisms as risk factors, but the results that known risk loci only account for a small proportion of risk are not encouraging for personal risk prediction based on genotyping [3]. This suggests other risk factors possibly affect the development of CVD between genetics and environmental factors, such as epigenetics.

Epigenetics is based on stable and heritable alterations in gene expression without changes in the DNA sequence itself [4]. DNA methylation is a crucial type of epigenetic modification, by which a methyl group covalent bonds to the $\mathrm{C} 5$ position of cytosine in $5^{\prime}$-C-phosphate-G- $3^{\prime}$ (CpG) dinucleotides, ultimately regulating the gene transcription activity and alter biological functions [5]. Previous studies have demonstrated associations of DNA methylation with atherosclerosis [6, 7], ischemic heart disease [8], coronary heart disease (CHD) [8], coronary artery disease [9] and acute coronary syndrome (ACS) [10]. Besides, DNA methylation is suggested as a biomarker for CVD risk [11]. However, the previous evidences were based on cross-sectional studies, lack of the rationale of temperal causality. Systemic identification of genome-wide DNA differential CpGs and analysis of their association with CVD risk are lacking.

In the present study, our main objective is to identify CpGs associated with the risk of CVD and further investigate their potential predictive value in CVD development for high-risk subjects. We first examined the association of genome-wide DNA methylation patterns from whole blood samples in 83 pairs of case-control participants at high-risk of CVD using Infinium Human Methylation EPIC Beadchip (850 K). For identified significant $\mathrm{CpGs}$, we further validated their association with the development of CVD in another independent cohort. And enrichment analysis in Gene Ontology (GO) terms was conducted to identify significantly enriched categories. Finally, we performed the ROC analysis to assess the predictive value of genome-wide significant $\mathrm{CpGs}$ for the risk of CVD.

\section{Methods}

\section{Study design and study samples}

The study samples were collected from the China PEACE Million Persons Project (MPP) cohort. The design and conduct of the project pilot have been previously described in detail [12]. In brief, the ChinaPEACE MPP is a national, population-based screening project funded by the Chinese government to identify individuals at high-risk of CVD. The project enrolled community population if they were $35-75$ years old and could confirm their residence in a selected region including 141 primary health care sites (88 rural counties and 53 urban districts) from all 31 provinces in the mainland of China from September 15, 2014 to June 20,2017 . The participants were initially screened for high-risk of CVD by measurements of blood pressure, height, weight and blood lipid, and a questionnaire on cardiovascular-related health status. The subjects at high-risk of CVD were identified if they met at least one of four criteria: 1 . Medical history of myocardial infarction (MI), percutaneous coronary intervention (PCI), coronary artery bypass grafting (CABG) treatment, or stroke; 2. High blood pressure defined as systolic blood pressure $(\mathrm{SBP}) \geq 160 \mathrm{mmHg}$ or diastolic blood pressure $(\mathrm{DBP}) \geq 100 \mathrm{mmHg}$; 3. Dyslipidemia defined as lowdensity lipoprotein cholesterol $(\mathrm{LDL}-\mathrm{C}) \geq 160 \mathrm{mg} / \mathrm{dL}$ $(4.14 \mathrm{mmol} / \mathrm{L})$ or high-density lipoprotein cholesterol $(\mathrm{HDL}-\mathrm{C})<30 \mathrm{mg} / \mathrm{dL}(0.78 \mathrm{mmol} / \mathrm{L})$; 4 . Risk of CVD in 10 years $\geq 20 \%$ based on WHO/ISH Cardiovascular Risk Prediction Charts for the Western Pacific Region B [13]. The high-risk participants received further health assessments, including electrocardiography, ultrasound scan, blood and urine analysis, and a questionnaire on lifestyle and medical history and were followed up every year to collect information on medication adherence, risk factor control, and any hospitalization. The data were collected from standardized in-person interviews by trained medical staff. The central ethics committee at the China National Center for Cardiovascular Disease (NCCD) approved this project. All participants had completed a written informed consent before participation in the project.

In the present study, we performed a nested case-control study based on the high-risk participants from the MPP cohort to examine and validate the association of genome-wide CpGs with the development of CVD. The inclusion criteria: 1 . Blood samples were collected after January 1, 2016; 2. Two years follow-up data was available; 3 . The questionnaire for the high-risk participants was available. Patients were excluded if they have selfreported any of medical history of cancer, chronic liver and kidney disease, infectious diseases, or any CVD at baseline.

Among the above eligible population, case (subjects who developed cardiovascular events) and control (subjects who did not develop cardiovascular events) were 1:1 matched for age (within 1 year), sex, BMI, the month of blood collection (within 1 month), medications treatment (antiplatelet medications and statins), ethnicity (han or non-han), current smoking status, and current alcohol intake at baseline. Then, 121 pairs were randomly sampled from the above eligible case-control pairs. Among 
them, 83 pairs case-control and 38 pairs case-control were randomly selected and regarded as the discovery set and the replicated set, respectively. Additional file shows this in more detail. [see Additional file 2: Figure S1].

\section{Clinical variables}

Cardiovascular events were self-reported by participants during follow-up visits. In the present study, hypertension was defined as $\mathrm{SBP} \geq 140 \mathrm{mmHg}$, or $\mathrm{DBP} \geq 90 \mathrm{mmHg}$, or self-reported any antihypertensive drugs. Hyperlipidemia was defined as total cholesterol (TC) $\geq 6.2 \mathrm{mmol} / \mathrm{L}$ or LDL-C $\geq 4.1 \mathrm{mmol} / \mathrm{L}$ according to Chinese guideline for adult's blood lipid, or self-reported lipid-lowering drug. Diabetes was defined as blood glucose greater than $7 \mathrm{mmol} / \mathrm{L}$ after at least $8 \mathrm{~h}$ after last meal or random blood glucose greater than $11.1 \mathrm{mmol} / \mathrm{L}$, or any kind of hypoglycemic agents. Details of these variables are shown in Table 1.

In this study, TC, LDL-C, HDL-C, triglycerides (TG), and high-sensitivity $\mathrm{C}$-reactive protein were measured by standardized enzymatic methods using Beckman Coulter AU680 analyzers (Beckman AU reagent). Hemoglobin A1c (HbA1c) was measured by high-performance liquid chromatography on the Arkray ADAMS-A1C HA-8180 analyzer. All tests were completed at the NCCD laboratory.

\section{Clinical outcomes}

CVD events included CVD admission and CVD death. CVD admission was defined as any hospitalization for

Table 1 Participant characteristics

\begin{tabular}{|c|c|c|c|c|c|c|}
\hline \multirow[t]{2}{*}{ Variables } & \multicolumn{3}{|l|}{ Discovery set } & \multicolumn{3}{|l|}{ Replication set } \\
\hline & Cases $(n=83)$ & Controls $(n=83)$ & $p$ & Cases $(n=38)$ & Controls $(n=38)$ & $p$ \\
\hline \multicolumn{7}{|l|}{ Basic characteristic } \\
\hline Age & $62(8)$ & $62(8)$ & 0.986 & $61(9)$ & $61(9)$ & 1 \\
\hline Male & $47(56.6)$ & $47(56.6)$ & 1 & $20(52.6)$ & $20(52.6)$ & 1 \\
\hline Ethnicity (han) & $78(94.0)$ & $78(94.0)$ & 1 & $37(97.4)$ & $37(97.4)$ & 1 \\
\hline Current smoker & $26(31.3)$ & $26(31.3)$ & 1 & $11(28.9)$ & $11(28.9)$ & 1 \\
\hline Smoking inde $x^{a}$ & $820(490,940)$ & $545(305,860)$ & 0.180 & $570(320,860)$ & $620(480,880)$ & 0.804 \\
\hline Drinker & $11(13.3)$ & $11(13.3)$ & 1 & $4(10.5)$ & $4(10.5)$ & 1 \\
\hline BMI & $25.5(4.0)$ & $25.5(4.0)$ & 0.956 & $26.0(3.5)$ & $26.0(3.5)$ & 0.983 \\
\hline \multicolumn{7}{|l|}{ Diagnosis } \\
\hline Hyperlipidemia & $19(22.9)$ & $9(10.8)$ & 0.038 & $5(13.2)$ & $6(15.8)$ & 0.744 \\
\hline Hypertension & $79(95.2)$ & 76 (91.6) & 0.349 & $34(89.5)$ & $33(86.8)$ & 0.723 \\
\hline Diabetes & 32 (38.6) & $31(37.3)$ & 0.873 & $12(31.6)$ & $16(42.1)$ & 0.342 \\
\hline Family history of CHD & $6(7.2)$ & $3(3.6)$ & 0.304 & $0(0.0)$ & $2(5.3)$ & 0.152 \\
\hline \multicolumn{7}{|l|}{ Clinical exams } \\
\hline Systolic pressure & $164(21)$ & $163(20)$ & 0.553 & $165(22)$ & $158(21)$ & 0.137 \\
\hline Diastolic pressure & $95(13)$ & $91(12)$ & 0.019 & $96(13)$ & $91(13)$ & 0.150 \\
\hline $\mathrm{HbA1c}(\%)$ & $4.9(4.6,5.3)$ & $5.0(4.6,5.4)$ & 0.639 & $4.8(4.6,5.3)$ & $4.9(4.7,5.2)$ & 0.714 \\
\hline hsCRP (mg/L) & $0.8(0.5,2.1)$ & $1.1(0.5,2.4)$ & 0.317 & $1.2(0.7,2.4)$ & $1.1(0.6,2.3)$ & 0.373 \\
\hline $\mathrm{HDL}-\mathrm{C}(\mathrm{mmol} / \mathrm{L})$ & $0.8(0.7,1.0)$ & $0.8(0.7,1.1)$ & 0.981 & $0.9(0.7,1.1)$ & $0.9(0.7,1.0)$ & 0.593 \\
\hline $\mathrm{LDL}-\mathrm{C}(\mathrm{mmol} / \mathrm{L})$ & $2.6(2.1,3.2)$ & $2.4(2.0,3.0)$ & 0.188 & $2.6(2.1,3.0)$ & $2.6(2.2,3.3)$ & 0.461 \\
\hline $\mathrm{TG}(\mathrm{mmol} / \mathrm{L})$ & $1.3(0.9,1.9)$ & $1.1(0.8,1.6)$ & 0.223 & $1.3(1.0,1.7)$ & $1.5(0.9,2.0)$ & 0.262 \\
\hline $\mathrm{TC}(\mathrm{mmol} / \mathrm{L})$ & $4.3(3.6,5.0)$ & $4.2(3.5,5.1)$ & 0.549 & $4.2(3.7,5.0)$ & $4.4(3.7,5.5)$ & 0.424 \\
\hline \multicolumn{7}{|l|}{ Events during follow up } \\
\hline CVD death ${ }^{b}$ & $18(21.7)$ & 0 & NA & $5(13.2)$ & 0 & NA \\
\hline CVD admission ${ }^{c}$ & $65(78.3)$ & 0 & NA & $33(86.8)$ & 0 & NA \\
\hline AMI & $24(28.9)$ & 0 & NA & $4(10.5)$ & 0 & NA \\
\hline Ischemic stroke & $41(49.4)$ & 0 & NA & $29(76.3)$ & 0 & NA \\
\hline
\end{tabular}

a The smoking index was calculated based on current smokers, Smoking index = the number of cigarettes smoked per day * years of smoking

${ }^{\mathrm{b}} \mathrm{CVD}$ death was defined as death due to $\mathrm{Ml}$, angina, heart failure, ischemic or hemorrhagic stroke

${ }^{\mathrm{C}} \mathrm{CVD}$ admission is defined as any hospitalization for $\mathrm{MI}$, ischemic stroke, $\mathrm{CABG}, \mathrm{PCl}$, and thrombolysis treatment for acute $\mathrm{MI}$ or stroke. $\mathrm{CHD}$, coronary heart disease; $\mathrm{BMI}$, body mass index; AMI, acute myocardial infarction; CVD, cardiovascular disease; HbA1c, hemoglobin A1c; hsCRP, high sensitivity C reactive protein; HDL-C, high density lipoprotein cholesterol; LDL-C, low density lipoprotein cholesterol; TG, triglyceride; TC, total cholesterol 
MI, ischemic stroke, CABG, PCI, and thrombolysis treatment for acute MI or stroke. CVD death was defined as death due to MI, angina, heart failure (HF), ischemic or hemorrhagic stroke reported by patient's relatives or doctors. For the patients who have developed multiple cardiovascular events, only the earliest one was used in subsequent analyses.

\section{DNA methylation analysis}

Genomic DNA was isolated from human whole blood samples using Chemagic 360 and chemagic DNA Buffy Coat 200 Kit according to the manufacturer's protocol. The quality and quantity of the extracted genomic DNA was analyzed with a DropSense 96 Spectrophotometer. The samples (500 ng genomic DNA) were treated for sodium bisulfite conversion using the EZ DNA methylation Gold Kit (Zymo Research, USA). And Genomewide DNA methylation profiles were assessed using the Infinium Human Methylation EPIC BeadChip (850 K) (Illumina, USA) following manufacturers' protocol, and DNA methylation level was quantified as a $\beta$ value. $\beta$ value represents the ratio of the fluorescent signal intensity measured by methylated and unmethylated probes and range from 0 (all copies of the CpG site in the sample are unmethylated) to 1 (all copies of the CpG site in the sample are methylated) [14].

Data quality control was performed using the chip analysis methylation pipeline (ChAMP) package [15] implemented in $R$ both in discovery set and in replication set. Probes meeting one of following criteria were removed: (1) probes with detection $p>0.01$; (2) probes with $<3$ beads in at least $5 \%$ of samples; (3) all non-CpG probes contained in dataset; (4) all multi-hit probes; (5) probes containing single nucleotide polymorphisms [16]; and (6) all probes located on chromosome X and Y. After quality control, 769,031 CpGs were retained for analysis. The filtered data were normalized by Beta Mixture Quantile dilation (BMIQ) [17]. Additionally, batch effect was adjusted to reduce technical biases by COMBAT, which was evident in a singular value decomposition (SVD) analysis [18].

\section{Statistical analysis}

Differences in baseline characteristics between case and control groups were evaluated by Kruskal-Wallis test for continuous variables or Chi-Square test for categorical variables. Before genome-wide DNA methylation association analysis, we performed principal component analysis (PCA) using 769,031 CpGs in both discovery and replication set, and the first 5 principal components (PCs) with proportion of variance $>1 \%$ were included as covariates in model for minimizing the potential technical bias. To investigate the association of CVD outcomes and DNA methylation level at each $\mathrm{CpG}$, we fitted linear regression models using $\mathrm{CpGassoc}$ package in $R$, with $\beta$ as dependent variables and CVD outcomes as independent variables. The models were adjusted for covariates, including age, sex, BMI, current smoking status, hypertension, diabetes mellitus, hyperlipidemia, cell components and the first 5 PCs. Cell components were estimated by the proportions of whole blood $(\mathrm{CD} 4+\mathrm{T}$ cells, CD8 $+\mathrm{T}$ cells, B cells, NK cells, monocytes, and neutrophile granulocyte) using the minfi [19] of $R$ package based on Houseman approach. We used the FDR $<0.005$ to identify significant CVD-associated CpGs in discovery set.

In replication set, the significant CpGs identified in discovery set were validated by linear regression models using CpGassoc package in $R$. The models were adjusted for the same factors as that in discovery set. The CpGs with $p<0.05$ in replication set and the consistent direction of effect in both discovery and replication sets were considered as significant replication CpGs.

For significant $\mathrm{CpGs}$, we used the annotation provided by Illumina and University of California Santa Cruz UCSC database (GRCh37/hg19). The genes annotated to CpGs with $p<10^{-4}$ in discovery set were selected for the enrichment analysis. The enrichment analysis in GO terms were conducted with $\mathrm{R}$ package 'clusterProfiler', and FDR $<0.05$ was used to identify significantly enriched categories.

Furthermore, ROC analysis was performed to assess the predictive value of significant CpGs in CVD outcomes. There were two prediction models included in ROC analysis: 1) model with baseline characteristics only; 2) model with both baseline characteristics and the significant CpGs. As a reference model, baseline characteristics included age, gender, BMI, current smoking status, current alcohol intake, hypertension, diabetes mellitus, hyperlipidemia, antiplatelet medication, stains treatment and family history of coronary heart disease. Statistical analysis were performed using the SAS version 9.4 and $\mathrm{R}$ version 3.5 .

\section{Results}

\section{Characteristics of the study subjects}

The baseline characteristics of the study subjects are shown in Table 1. In discovery set, there were 83 CVD cases (65 admissions for CVD, 18 died of CVD) and 83 controls with mean age of 62 years. The replication set included 38 CVD cases (33 admissions for CVD, 5 died of CVD) and 38 controls with mean age of 61 years. As the study subjects were from the high-risk participants of the MPP cohort, the proportion of hypertension subjects in our study cohort was approximately as high as $90 \%$. There was no significant difference in most variables 
between the case and control group in two cohorts, except for hyperlipidemia $(p=0.038)$ and diastolic pressure $(p=0.019)$ in the discovery set.

\section{Epigenome-wide association analysis}

Before performing a epigenome-wide association analysis, we did SVD analysis and PCA based on plate number, chip number and date of test for examining batch effect in the normalized data by BMIQ and the further processed data by COMBAT, respectively. SVA analysis showed that batch effect was almost completely controlled after adjustment in different chips and different sample locations by COMBAT [see Additional file 2: Figure S2, S3]. In the PCA, no significant aggregations were found between different plates, different chips and different date of test (see Additional file 2: Figure S4). After that, PCA based on study samples was conducted using 769,031 CpGs in both discovery and replication set, and the first 5 PCs with proportion of variance $>1 \%$ were included as covariates in the genome-wide association analysis by linear regression model [see Additional file 2: Table S1, S2]. The results showed that eight $\mathrm{CpGs}$ achieved a genome-wide significance level $(F D R<0.005)$ after adjustment for age, gender, BMI, current smoking status, hypertension, diabetes mellitus, hyperlipidemia, cell compositions and the first 5 PCs (Fig. 1 and Table 2). Six of 8 CVD-associated CpGs were located in SPON1 gene body (cg11651314), in PACS1 gene body (cg03914662), in TSS200 of the UACA gene (cg09306458), in CCDC50 gene body ( cg05946546), in 1stExon of the CYP8B1 gene ( $\operatorname{cg} 07655795)$, and in HSD17B11 gene body ( cg02518222), respectively (Table 2). Of them, increased methylation level at CpG cg07655795 (CYP8B1) was associated with an increased risk of CVD, while decreased methylation level at the remaining $7 \mathrm{CpGs}$ were associated with an increased risk of CVD.

For the 8 CVD-associated CpGs $(F D R<0.005)$ identified in discovery set, we performed replication analysis in an independent cohort (38 cases and 38 controls). Two CpGs of them (cg09306458, cg06901278) were replicated $(p<0.05)$ with consistent direction of effect in both sets (Table 2). In two replicated CpGs, cg09306458 was located in regulatory region of the $U A C A$ gene, and cg06901278 had no annotation gene.

\section{Enrichment analysis}

Go term enrichment analysis were conducted to identify the possible functions affected by annotation genes. A total of 787 individual genes from 1036 CpGs with $p<10^{-4}$ in discovery set were used for enrichment analysis. There were 17 significantly functional categories identified $(\mathrm{FDR}<0.05)$, including 6 categories in biological processes (BP), and 11 categories in cellular component (CC) (Fig. 2) (see Additional file 1: Excel S1). The most enriched categories were significant enrichment for anatomical structure homeostasis as well as regulation
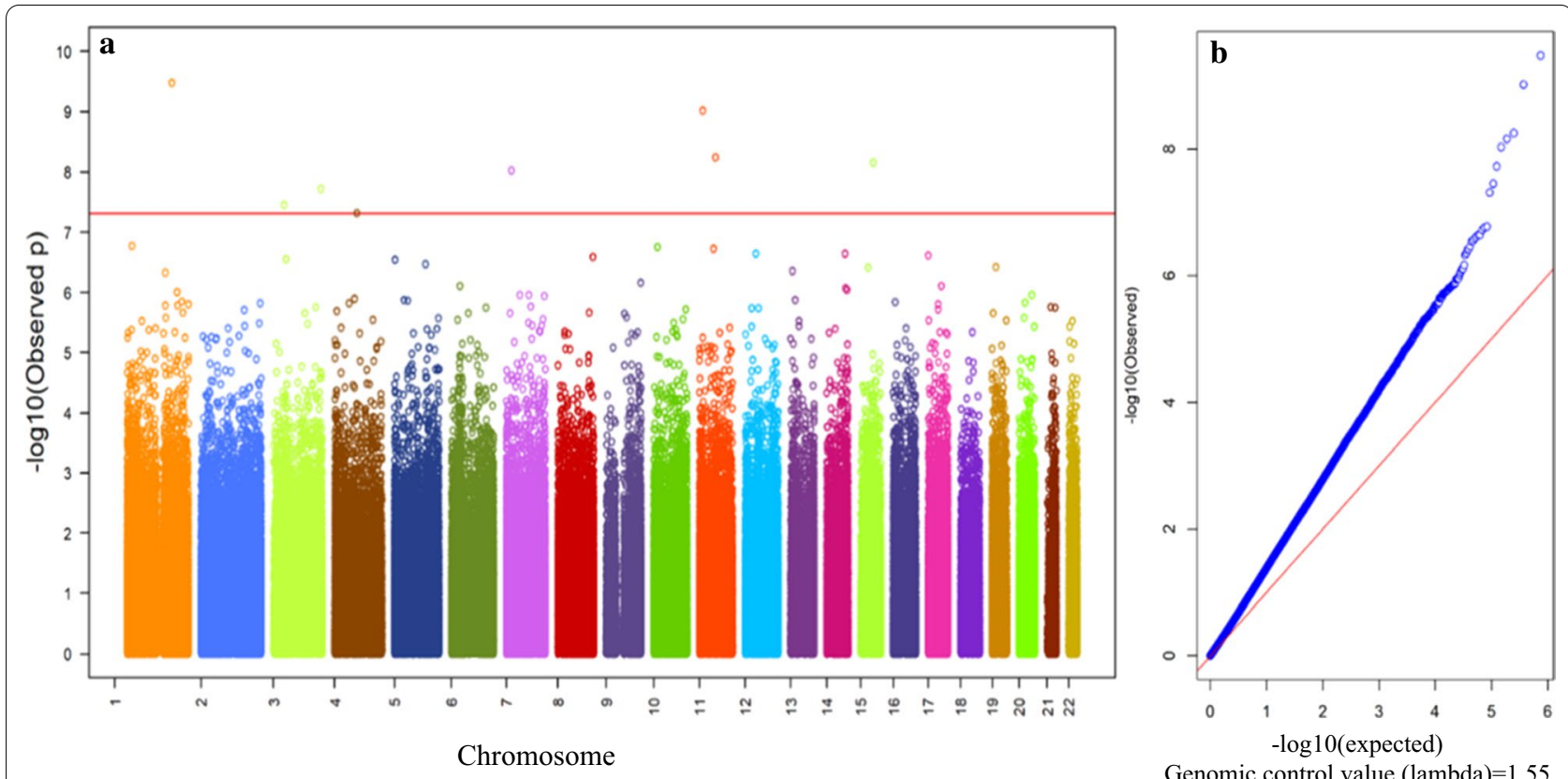

Fig. 1 Manhattan plot (a) and QQ plot (b) in the discovery set. In the Manhattan plot, red line represents - $\log 10 P$ at false discovery rate $(F D R)=0.005$ 
Table 2 Association analysis for CVD outcomes with genome-wide significant CpGs identified in the discovery set (FDR <0.005)

\begin{tabular}{|c|c|c|c|c|c|c|c|c|c|}
\hline \multirow[t]{2}{*}{ CpG } & \multirow[t]{2}{*}{ Gene } & \multirow[t]{2}{*}{ Relation to gene } & \multicolumn{4}{|c|}{ Discovery set } & \multicolumn{3}{|c|}{ Replication set } \\
\hline & & & Effect* & SE & $p$ & FDR & Effect & SE & $p$ \\
\hline cg06901278 & - & - & -0.022 & 0.003 & $3.37 \mathrm{E}-10$ & $<0.001$ & -0.015 & 0.006 & 0.010 \\
\hline $\operatorname{cg} 11651314$ & SPON1 & Body & -0.033 & 0.005 & $9.70 E-10$ & $<0.001$ & -0.012 & 0.007 & 0.072 \\
\hline cg03914662 & PACS1 & Body & -0.0177 & 0.003 & $5.73 \mathrm{E}-09$ & 0.0013 & -0.004 & 0.005 & 0.415 \\
\hline cg09306458 & UACA & TSS200 & -0.009 & 0.001 & 7.01E-09 & 0.0013 & -0.005 & 0.002 & 0.016 \\
\hline cg05359217 & - & - & -0.012 & 0.002 & $9.34 \mathrm{E}-09$ & 0.0014 & -0.002 & 0.003 & 0.591 \\
\hline cg05946546 & CCDC50 & Body & -0.017 & 0.003 & $1.88 \mathrm{E}-08$ & 0.0024 & -0.003 & 0.005 & 0.538 \\
\hline cg07655795 & CYP8B1 & 1stExon & 0.024 & 0.004 & $3.55 E-08$ & 0.0039 & 0.007 & 0.006 & 0.249 \\
\hline cg02518222 & HSD17B11 & Body & -0.042 & 0.007 & $4.85 E-08$ & 0.0047 & -0.017 & 0.011 & 0.111 \\
\hline
\end{tabular}

TSS, transcription start site; FDR, false discovery rate. *Effect value represents change in $\beta$ value per change from control to case

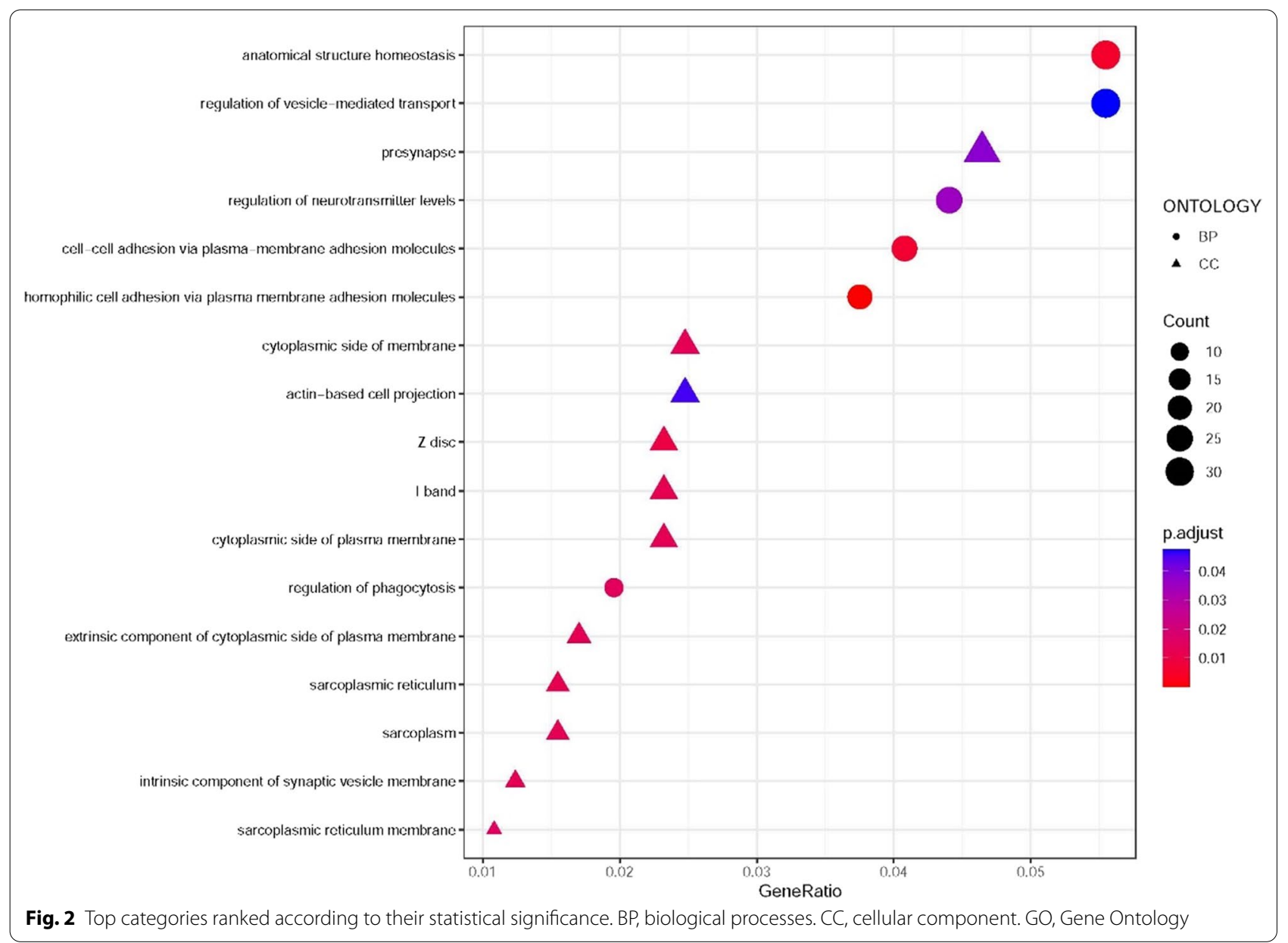

of vesicle-mediated transport, which were located in BP group (Fig. 2).

\section{ROC analysis}

ROC curves were drawn to assess the specificity and sensitivity of CVD prediction on the basis of $8 \mathrm{CVD}$-related
CpGs identified in discovery set and baseline characteristics as a reference model. As shown in Fig. 3a, the model with both 8 CVD-related CpGs and baseline characteristics showed an excellent predictive effect for CVD occurrence and much better than the model with baseline characteristics only $(\mathrm{AUC}=0.967,95 \% \mathrm{CI}$ 


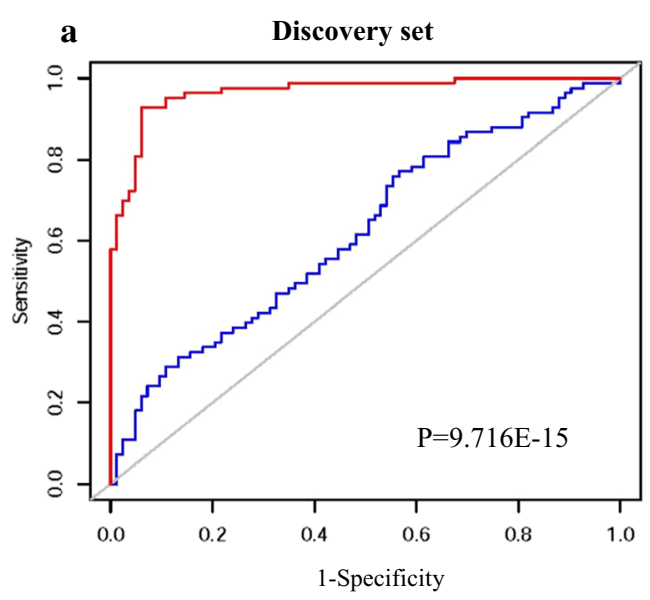

- Model combined 8 CpGs with clinical characteristics, AUC:0.967 (0.942-0.991)

- Model with clinical characteristics, AUC:0.621 (0.536-0.706) b

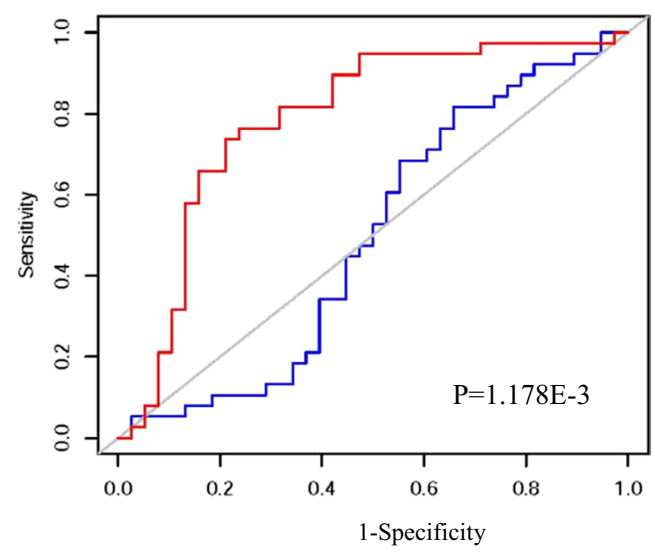

- Model combined 8 CpGs with clinical characteristics, AUC:0.768 (0.676-0.896)

- Model with clinical characteristics, AUC:0.492 (0.357-0.628)

Fig. 3 Receiver operating characteristic (ROC) analysis of the sensitivity and specificity of CVD prediction by the model combined CVD-related CpGs and baseline characteristics. $\mathbf{a}$ indicates the discovery set; $\mathbf{b}$ indicates the replication set. AUC, area under the curve. The baseline characteristics were assessed as a reference model, including age, gender, BMI, current smoking status, current alcohol intake, hypertension, diabetes mellitus, hyperlipidemia, antiplatelet medication, stains treatment and family history of coronary heart disease

(0.942-0.991); AUC $=0.621,95 \%$ CI $(0.536-0.706)$; $p=9.716 \mathrm{E}-15)$. In replication set, we also found the similar trend that the model combined 8 CVD-related CpGs and baseline characteristics showed significant predictive capacity compared with the reference model with baseline characteristics only (AUC $=0.786,95 \% \mathrm{CI}$ (0.676-0.896); $\mathrm{AUC}=0.492,95 \%$ CI $(0.357-0.628)$; $P=1.178$ E-3) (Fig. 3b).

\section{Discussion}

The present study provides evidence that DNA methylation level in whole blood DNA was associated with the risk of CVD at specific CpGs in high-risk Chinese population. In the discovery analysis, we identified 8 significant CVD-related CpGs after adjustment for conventional covariates and the first 5 PCs. And two of these CVD-associated CpGs were replicated in an independent cohort. Enrichment analysis revealed that the genes annotated to CpGs with $p<10^{-4}$ in discovery set were related with significant enrichment for anatomical structure homeostasis as well as regulation of vesicle-mediated transport. ROC analysis suggested that CVD-associated CpGs identified could provide additional predictive power for CVD occurrence in high-risk subjects.

In this study, there were some strengths in minimizing the potential confounding effect to ensure the findings reliable. First, the case-controls were strictly matched for the traditional risk factors of CVD and factors probably affecting DNA methylation status. Second, normalized methylation data was processed for reducing batch effect by COMBAT, and examined by SVD analysis and PCA. Third, we have adjusted for age, sex, BMI, cell compositions, hypertension, diabetes mellitus, hyperlipidemia, and PCs in regression model. Fourth, our prospective design fits a temporal relationship between the DNA methylation level and CVD outcomes, which elucidated the causal relationship more reasonablly. In addition, Infinium Human Methylation EPIC Beadchip (850 K) almost doubled the number of CpGs on the $450 \mathrm{~K}$ array which was widely used in previous studies, which improves coverage within intergenic regions, enhancers, and distal regulatory elements [20]. In view of the above reasons, we believe that our results are reliable although some degree of inflation in the QQ-plot $(\lambda=1.55)$ was observed in our study, which might be due to the polygenic effect of DNA methylation on CVD or the residual confounding that was not completely controlled in the linear regression models.

In discovery phase, there were 8 CpGs significantly associated with CVD development in the Chinese population. The CpGs cg09306458 was located in regulatory region of the UACA gene, which encodes a protein that contains ankyrin repeats and coiled coil domains and likely plays a role in apoptosis. A study of the Danish National Birth Cohort (608 cases vs 626 control) identified the UACA gene at the cg12157761 associated with the offspring of women with gestational diabetes mellitus [21]. In addition, a meta-analysis showed that newborn 
blood DNA CpG cg01244124 located in UACA was negatively associated with sustained maternal smoking during pregnancy [22]. Though the gene $U A C A$ in responding to different $\mathrm{CpGs}$ among the various studies, the results of these two studies were not contradictive with our findings, in that diabetes mellitus and smoking were the classic risk factor of CVD and may induce DNA methylation alteration at specific site to result in the outcome of CVD.

The CpGs cg11651314 located in the gene body region of SPON1 was also associated with CVD development at genome-wide level in our study. SPON1 encodes the developmentally regulated protein F-spondin, which has been reported to be a putative ligand for the amyloid precursor protein [23]. We observed decreased methylation at this CpGs, which was associated with the increased risk of CVD, and a previous study for DNA methylation in adipose tissue also showed SPON1 in corresponding to the site cg23284931 was negatively correlated with type 2 diabetes [24]. Additionally, proteomic profiling study in community-based prospective cohorts revealed that higher levels of circulating protein SPON1 at baseline was associated with worsened systolic function and HF incidence independently of established risk factors [25]. So it is likely that the hypomethylated status could enhance the expression of SPON1 and contribute to the pathogenesis of CVD. However, in the atherosclerotic human aorta sample, SPON1 was significantly hypermethylated compared with the plaque-free intima [26], reflecting the difference of DNA methyaltion pattern at certain genes in different tissues.

The hypermethylated CpGs cg07655795 identified in EWAS was located in the 1stExon region of CYP8B1 (Cytochrome P450 Family 8 Subfamily B Member 1). CYP8B1 was one of the key enzymes of bile acids synthesis, and ablating CYP8B1 in mice led to reduced absorption of dietary triglyceride with intact triglyceride hydrolysis and improved insulin sensitivity, suggesting CYP8B1 as a potential therapeutic target for obesity and diabetes [27]. Another significant CpG cg07655795 associated with CVD risk in our study was located in body region of gene HSD17B11 (Hydroxysteroid $17-\beta$ Dehydrogenase 11$)$. In a integrated microarray analysis, HSD17B11 was one of the up regulated genes in coronary artery disease, and pathway enrichment analysis revealed that differentially expressed genes in coronary artery disease were mostly enriched in the superpathway of steroid hormone biosynthesis, and so on [28]. In addition, our findings showed that CpGs cg03914662 and cg05946546 were located in the body region of PACS1 and $C C D C 50$, respectively. PACS1 encodes phosphofurin acidic cluster sorting protein 1 , which is a multifunctional membrane traffic regulator that plays an important role in organ homeostasis [29]. CCDC50 encodes Ymer, an effector of epidermal growth factor (EGF)-mediated cell signaling that is ubiquitously expressed in different organs and has been suggested to inhibit down-regulation of the EGF receptor [30,31]. Up to date, no evidence has associated DNA methylation with CVD or CVD risk factor in regard to PACS1 and CCDC50. Therefore, it's essential to further validate the CpG site $\operatorname{cg} 03914662$ and cg05946546 in a larger independent cohort and assess the association with the expression of its annotation genes.

There were no overlap between our identified CVDrelated CpGs and the sites reported in previous MI-associated $[32,33]$ and ACS-associated EWAS studies ${ }^{[34]}$ with whole blood sample. Rask-Andersen et al. [32] identified 211CpGs mapped to 196 annotation genes in individuals with a history of MI from northern Sweden population, and 42 genes among them had been described to be related to cardiac function, CVD, cardiogenesis and recovery after ischemic episode. Another study identified 3 CpGs cg07786668 in ZFHX3, cg17218495 in SMARCA4 and cg06642177 in SGK1 that significantly associated with MI in Japan [33]. Li Jun and colleagues [34] identified 47 ACS-associated CpGs annotated to 44 individual genes at both whole blood and cell level in 102 ACS patients and 101 controls from Chinese population. This lack of overlap between our CVD-related CpGs and MI- or ACS-associated CpGs may be due to the following reasons: (i) Our prospective cohort study is different from the cross-sectional cohort in other studies; (ii) Infinium Human Methylation EPIC Beadchip (850 K) has a larger number of CpGs compared with the $450 \mathrm{~K}$ array widely used in previous studies; (iii) The CVD outcomes in our study were not exactly the same as the previous studies. However, our ROC analysis revealed that the model combined genome-wide CVD-associated CpGs with baseline characterastics provided significant predictive capacity for CVD development in high-risk population. Therefore, our identified CpGs may serve as potential biomarkers in clinical risk assessment for CVD.

In present study, Go enrichment ananlysis showed that the genes annotated to CpGs with $p<10^{-4}$ in discovery set were involved in anatomical structure homeostasis as well as regulation of vesicle-mediated transport that may suggest a complex impairment of cellular function [32] in the occurrence of CVD. However, none of 6 CVD-related annotated genes identified in discovery set was overlapped with the enriched genes in GO enrichment analysis. In complex diseases (e.g., cardiovascular disease), the contribution of most genes to the occurrence of disease is small, which may result in these genes not easy to be 
identified by EWAS, especially given the small sample size of this cohort. Therefore, these genes may not have been included in the enrichment analysis, although we have aleady included the genes annotated to CpGs with $p<10^{-4}$. This may be a reason why the results of enrichment analysis did not overlap with the main results of the study. Nevertheless, considering the good prediction effect in ROC analysis, we believe that it is promising to use these CpGs found in the study as potential biomarkers for predicting the occurrence of CVD.

The present study has some limitations. First, the sample size was small both in discovery and replication sets, so we should validate our results in a larger independent cohort. Second, we used whole blood samples to examine the DNA methylation level, which may not directly reflect the status of the target tissue. Third, we did not assess the association between gene expression and CpGs which would improve the confidence of the results. Moreover, self-reported medical history might introduce recall bias, although the data were collected by trained medical staff. Finally, our findings need further validation in other ethnicity groups and population with relatively low CVD risk.

\section{Conclusion}

In conclusion, DNA methylation level at 8 novel CpGs was associated with CVD development in a Chinese population, and two of them were replicated in another independent cohort. Enrichment analysis in discovery set revealed a significant enrichment for anatomical structure homeostasis and regulation of vesicle-mediated transport. ROC analysis showed that the model with both CVD-associated CpGs and baeline characterastics had excellent predictive capacity for CVD occurence in high-risk subjects. Future studies ought to verify the predictive effect of these CpGs on CVD in other ethnicity groups and population with relatively low CVD risk and further elucidate the potential functional mechanism of CVD-related CpGs.

\footnotetext{
Abbreviations

CVD: Cardiovascular disease; EWAS: Epigenome-wide association study; FDR: False discovery rate; ROC: Receiver operating characteristic; AUC: Area under the curve; GWAS: Genome-wide association studies; ACS: Acute coronary syndrome; MI: Myocardial infarction, PCI: Percutaneous coronary intervention; CABG: Coronary artery bypass grafting; SBP: Systolic blood pressure; DBP: Diastolic blood pressure; LDL-C: Low-density lipoprotein cholesterol; HDL-C: High-density lipoprotein cholesterol; NCCD: National Center for Cardiovascular Disease; TC: Total cholesterol; TG: Triglycerides; HF: Heart failure; PCs: Principal components; BMI: Body mass index; CHD: Coronary heart disease; AMI: Acute myocardial infarction; HbA1c: Hemoglobin A1c; hsCRP: High sensitivity C reactive protein; TSS: Transcription start site; UTR: Untraslated region; PCA: Principal component analysis; BMIQ: Beta Mixture Quantile dilation; SVD: Singular value decomposition; CHAMP: Chip analysis methylation pipeline.
}

\section{Supplementary information}

The online version contains supplementary material available at https://doi. org/10.1186/s12872-021-02001-w.

Additional file 1: Figures S1-S4 and Tables S1-S2. Figure S1. Flow chart of study sample selection. Figure $\mathbf{S 2}$. Singular value decomposition (SVD) analysis by data before processing by COMBAT. Figure S3. Singular value decomposition (SVD) analysis by data after processing by COMBAT. Figure S4. Principal component analysis (PCA) based on plate number (A) chip number (B), date of test (C) and gender (D). Table S1. The top 5 principal components (PCs) with proportion of variance more than 0.01 used for epigenomewide association analysis in the discovery set. Table S2. The top 5 principal components (PCs) with proportion of variance more than 0.01 used for epigenomewide association analysis in the replication set.

Additional file 2: Appendix 1-3. Appendix 1. Significantly functions categories in Gene Ontology (GO) enrichment analysis in discovery set. Appendix 2. Significant Differentially Methylated Regions (DMRs) with annotated genes by hump-hunting method in the discovery group. Appendix 3. Significant Differentially Methylated Regions (DMRs) with annotated genes by Probe Lasso method in the discovery group.

\section{Acknowledgments}

We appreciate the study team at the National Center for Cardiovascular Diseases for project design, collection of clinical information and blood samples. We are grateful for the funding support provided by the Chinese government.

\section{Authors' contributions}

Yan Gao and Huifang Pang interpreted the results and wrote the manuscript. Xin Zheng as the corresponding author and Yan Gao designed and implemented the study, interpreted the data, and reviewed the manuscript. Bowang Chen and ChaoQun Wu as statistician designed the biostatistical methods and analyzed the data. Yanping Wang and Dianjianyi Sun reviewed the manuscript. Libo Hou and Siming Wang completed the biochemical analysis of blood samples and the quality control of DNA extraction. All authors read and approved the final manuscript.

\section{Funding}

This project was supported by the National Key Research and Development Program (2016YFE0103800) from the Ministry of Science and Technology of China, CAMS Innovation Fund for Medical Sciences (2017-I2M-2-002), the Major Public Health Service Project from the Ministry of Finance and National Health and Family Planning Commission of China and the 111 Project (B16005) from the Ministry of Education of China.

\section{Availability of data and materials}

The China PEACE Million Persons Project is a major national program, and as the government policy stipulates, it is not permissible for the researchers to make the raw data publicly available at this time. All data genetated during this study are included in this manuscript and its supplementary information files.

\section{Declarations}

\section{Ethics approval and consent to participate}

The ethics committee at the China National Center for Cardiovascular Disease approved this project. All participants had completed a written informed consent before participation in the project. We confirm that all methods were carried out in the study in accordance with relevant guidelines and regulations.

\section{Consent for publication}

Not applicable.

\section{Competing interests}

The authors declare that they have no competing interests. 


\section{Author details}

${ }^{1}$ National Clinical Research Center for Cardiovascular Diseases, State Key Laboratory of Cardiovascular Disease, Chinese Academy of Medical Sciences and Peking Union Medical College, Fuwai Hospital, National Center for Cardiovascular Diseases, 167 Beilishi Road, Beijing 100037, People's Republic of China ${ }^{2}$ Department of Epidemiology and Biostatistics, School of Public Health, Peking University Health Science Center, Beijing, People's Republic of China.

Received: 18 November 2020 Accepted: 7 April 2021

Published online: 12 May 2021

\section{References}

1. Joseph PG, Pare G, Anand SS. Exploring gene-environment relationships in cardiovascular disease. Can J Cardiol. 2013;29(1):37-45.

2. Thom T, Haase N, Rosamond W, et al. Heart disease and stroke statistics-2006 update: a report from the American Heart Association Statistics Committee and Stroke Statistics Subcommittee. Circulation. 2006:113(6):e85-151.

3. Whitfield JB. Genetic insights into cardiometabolic risk factors. Clin Biochem Rev. 2014:35(1):15-36.

4. Wu SS, Lin X, Yuan LQ, et al. The role of epigenetics in arterial calcification. Biomed Res Int. 2015:2015:320849.

5. Razin A, Webb C, Szyf M, et al. Variations in DNA methylation during mouse cell differentiation in vivo and in vitro. Proc Natl Acad Sci USA. 1984;81(8):2275-9.

6. McLean CY, Bristor D, Hiller M, et al. GREAT improves functional interpretation of cis-regulatory regions. Nat Biotechnol. 2010;28(5):495-501.

7. Turunen MP, Aavik E, Yla-Herttuala S. Epigenetics and atherosclerosis. Biochem Biophys Acta. 2009:1790(9):886-91.

8. Baccarelli A, Wright R, Bollati V, et al. Ischemic heart disease and stroke in relation to blood DNA methylation. Epidemiology. 2010:21(6):819-28.

9. Deloukas P, Kanoni S, Willenborg C, et al. Large-scale association analysis identifies new risk loci for coronary artery disease. Nat Genet. 2013;45(1):25-33.

10. Rask-Andersen M, Martinsson D, Ahsan M, et al. Epigenome wide association study reveals differential DNA methylation in individuals with a history of myocardial infarction. Hum Mol Genet. 2016;25(21):4739-48.

11. Kim M, Long TI, Arakawa K, et al. DNA methylation as a biomarker for cardiovascular disease risk. PLoS ONE. 2010;5(3):e9692.

12. Lu J, Xuan S, Downing NS, et al. Protocol for the China PEACE (patientcentered evaluative assessment of cardiac events) million persons project pilot. BMJ Open. 2016;6(1):e010200.

13. World Health Organization/International Society of Hypertension. WHO/ ISH Risk prediction charts for $14 \mathrm{WHO}$ epidemiological sub-regions. Available at : https://www.who.int/ncds/management/WHO_ISH_Risk_Predi ction_Charts.pdf?ua=1. Accessed February 1, 2020.

14. de Lichtenfels FCLAJ, vanderPlaat DA, deJong K, et al. Long-term air pollution exposure, genome-wide dna methylation and lung function in the lifelines cohort study. Environ Health Perspect 2018, 126(2):027004.

15. Morris TJ, Butche LM, Feber A, et al. ChAMP: 450k Chip analysis methylation pipeline. Bioinformatics. 2014;30(3):428-30.

16. Zhou W, Laird PW, Shen H. Comprehensive characterization, annotation and innovative use of Infinium DNA Methylation BeadChip probes. Nucleic Acids Res. 2017;45(4):e22.

17. Teschendorff AE, Marabita F, Lechner M, et al. A beta-mixture quantile normalization method for correcting probe design bias in Illumina Infinium 450 k DNA methylation data. Bioinformatics. 2013;29(2):189-96.
18. Leek JT, Johnson WE, Parker HS, et al. The SVA package for removing batch effects and other unwanted variation in high-throughput experiments. Bioinformatics. 2012:28:882-3.

19. Aryee MJ, Jaffe AE, Corrada-Bravo H, et al. Minfi: a flexible and comprehensive bioconductor package for the analysis of Infinium DNA methylation microarrays. Bioinformatics. 2014;30(10):1363-9.

20. Pidsley R, Zotenko E, Peters TJ, et al. Critical evaluation of the Illumina MethylationEPIC BeadChip microarray for whole-genome DNA methylation profiling. Genome Biol. 2016;17(1):208.

21. Hjort L, Martino D, Grunnet LG, et al. Gestational diabetes and maternal obesity are associated with epigenome-wide methylation changes in children. JCl Insight 2018, 3(17).

22. Sikdar S, Joehanes R, Joubert BR, et al. Comparison of smoking-related DNA methylation between newborns from prenatal exposure and adults from personal smoking. Epigenomics. 2019;11(13):1487-500.

23. Hoe H-S, Rebeck GW. Regulated proteolysis of APP and ApoE receptors. Mol Neurobiol. 2008;37(1):64-72.

24. Rodriguez-Rodero S, Menendez-Torre E, Fernandez-Bayon G, et al. Altered intragenic DNA methylation of HOOK2 gene in adipose tissue from individuals with obesity and type 2 diabetes. PLOS ONE. 2017;12(12):e0189153.

25. Stenemo M, Nowak C, Byberg L, et al. Circulating proteins as predictors of incident heart failure in the elderly. Eur J Heart Fail. 2018;20(1):55-62.

26. Yamada $Y$, Horibe $H$, Oguri M, et al. Identification of novel hyper- or hypomethylated $\mathrm{CpG}$ sites and genes associated with atherosclerotic plaque using an epigenome-wide association study. Int J Mol Med. 2018:41(5):2724-32

27. Bertaggia E, Jensen KK, Castro-Perez J, et al. Cyp8b1 ablation prevents western diet-induced weight gain and hepatic steatosis because of impaired fat absorption. Am J Physiol Endocrinol Metab. 2017:313(2):121-33.

28. Balashanmugam MV, Shivanandappa TB, Nagarethinam S, et al. Analysis of differentially expressed genes in coronary artery disease by integrated microarray analysis. Biomolecules. 2019;10(1):35.

29. Thomas G, Aslan JE, Thomas L, et al. Caught in the act: protein adaptation and the expanding roles of the PACS proteins in tissue homeostasis and disease. J Cell Sci. 2017;130(11):1865-76.

30. Blagoev B, Ong SE, Kratchmarova I, et al. Temporal analysis of phosphotyrosine-dependent signalling networks by quantitative proteomics. Nat Biotechnol. 2004:22(9):1139-45.

31. Kratchmarova I, Blagoev B, Haack-Sorensen M, et al. Mechanism of divergent growth factor effects in mesenchymal stem cell differentiation. Science. 2005;308(5727):1472-7.

32. Nakatochi M, Ichihara S, Yamamoto $K$, et al. Epigenome-wide association of myocardial infarction with DNA methylation sites at loci related to cardiovascular disease. Clin Epigenetics. 2017;9:54

33. Rask-Andersen M, Martinsson D, Ahsan M, et al. Epigenome-wide association study reveals differential DNA methylation in individuals with a history of myocardial infarction. Hum Mol Genet. 2016;25(21):4739-48.

34. Li J, Zhu X, Yu K, et al. Genome-wide analysis of DNA methylation and acute coronary syndrome. Circ Res. 2017;120(11):1754-67.

\section{Publisher's Note}

Springer Nature remains neutral with regard to jurisdictional claims in published maps and institutional affiliations. 